\section{SP1-60 COMMUNITY VIOLENCE AND THE PREVALENCE OF ASTHMA IN THE PERIPHERAL AREAS OF SALVADOR}

doi:10.1136/jech.2011.142976n.37

G da Costa Alves, * D N Santos, C A Feitosa, M L Barreto. Institute of Collective Health, Federal University of Bahia, Salvador, Bahia, Brazil

Introduction Asthma and violence are two complex, multi-factorial phenomena strongly present in Latin America and Brazil. Studies about asthma demonstrate that there are a number of risk factors for the development of the disease; among these are: poverty, diet and psycho-social factors. Violence, in particular community violence, is a significant psycho-social factor.

Objective To identify the degree of community violence reported by carers of children between 4 and 12 years old and the influence of this exposure on the occurrence of asthma symptoms in childhood.

Methods A transversal study was undertaken with 1232 carers resident in 24 areas of the city of Salvador. We investigated asthma symptoms in children and acts of violence in the community.

Results More than $75 \%$ of carers had been victims of community violence over the last year, with $20 \%$ indicating high degrees of coexistence with this phenomenon. Children exposed to the maximum degree of violence presented greater prevalence of asthma symptoms, peaking at $28.4 \%$, compared to $16.4 \%$ in non-exposed children. Children whose carers are exposed to the maximum degree of violence were almost twice as likely to present with these symptoms ( $\mathrm{OR}_{\mathrm{ajs}}{ }^{*} 1.94$ 95\% CI 1.12 to 3.36$)$.

Conclusion Exposure to community violence demonstrated a strong association with increase in the prevalence of asthma symptoms, highlighting the importance of this psychosocial risk factor in the manifestation of this disease.

\section{SP1-61 EPIDEMIOLOGICAL TREND IN TUBERCULOSIS (TB) IN ROMANIA BETWEEN 1990 AND 2008}

doi:10.1136/jech.2011.142976n.38

A L Golli, ${ }^{*}$ C Didilescu, M Nitu, M Olteanu, L Mitrache. University of Medicine and Pharmacy, Craiova, Romania

Background The epidemiology of tuberculosis (TB) in Romania highlights the need for early detection and directly observed therapy. This study analysed the evolution of the TB epidemic in Romania from 1990 to 2008 in order to evaluate the TB control strategy.

Methods A retrospective study describing the evolution of the main epidemiological indicators for TB in Romania, 1990-2008 was carried out. Incident TB cases were obtained from "Marius Nasta" Institute of Pulmonology from Bucharest, Ministry of Public Health Report 2008. Comparative European data were obtained from the European Health for All Database (HFA-DB) 2010 and WHO TB Report 2010.

Results The overall incidence of TB in Romania steadily declined from 142.2 per 100000 in 2002 to 107.8 per 100000 in 2008 at which time it was almost eight times higher than EU average (14.1 per $100000)$; the fourth highest national incidence of TB in the WHO Europe Region. Of the 21724 registered TB cases (incident and relapses) in 2008, 61\% were smear-positive; of these 9511 were incident cases. The incidence in the paediatric population was 12.7 per 100000 in 1990, peaking at 42 per 100000 in 2000 before falling to 28.7 per 100000 in 2008 . Mortality from TB has progressively fallen from 12 per 100000 in 1990 to 7.2 per 100000 in 2008 . In total $2.8 \%$ of all incident and $11 \%$ of all re-treatment cases in Romania were MDR TB cases in 2007, compared to $10 \%$ and $43 \%$ respectively in Europe. In 2008, 816 MDR TB cases were registered; 130 incident and 686 re-treatment cases.
Conclusions $A$ reduction in the incidence of and mortality from TB since 2002 confirms the effectiveness of measures to control TB.

\section{SP1-62 ROSE ANGINA VS SILENT ISCHAEMIA IN PREDICTION OF CORONARY HEART DISEASE: TEHRAN LIPID AND GLUCOSE STUDY}

doi:10.1136/jech.2011.142976n.39

${ }^{1} \mathrm{~N}$ Fahimfar, ${ }^{*} \mathrm{D}$ Khalili, ${ }^{1} \mathrm{G}$ Shafiee, ${ }^{1} \mathrm{~F}$ Hadaegh, ${ }^{2} \mathrm{~F}$ Azizi. ${ }^{1}$ Prevention of Metabolic Disorders Research Center, Research Institute for Endocrine Sciences, Shahid Beheshti University of Medical Sciences, Tehran, Iran; ${ }^{2}$ Endocrine Research Center, Research Institute for Endocrine Sciences, Shahid Beheshti University of Medical Sciences, Tehran, Iran

Introduction Coronary heart disease (CHD) is one of the most common causes of morbidity and mortality in Iran. The aim of this cohort study is evaluating the ability of Rose questionnaire (WHO angina questionnaire) and ECG to predict 10-year CHD events in a population with high prevalence of CHD

Methods Study population included 5652 subjects $\geq 30$ years ( 3244 females), free of self reported CHD at the baseline. Subjects were categorised to four groups according to their Rose Angina and ECG status (Whitehall criteria, Minnesota codes of 1.1, 1.2, 1.3, 1.4-4.4, $5.1-5.3,7.1$ in ECG) at the baselime as Rose-/ECG-, Rose +/ECG-, Rose-/ECG+, Rose+/ECG+. RR of CHD were estimated using Cox proportional hazard model given Rose-/ECG- as the reference.

Results 387 CHD events (169 women) were observed. After age adjustment, RR was 2.66 (95\% CI 1.76 to 4.01 ) and 2.50 (1.65 to 3.81) for Rose+/ECG - group in men and women respectively. These figures for Rose-/ECG + were 1.63 (1.09 to 2.45) and 2.21 (1.48 to 3.29). After more adjustment for traditional risk factors, RR was 2.60 (1.72 to 3.93) and 2.28 (1.50 to 3.47) for Rose+/ECG- in men and women respectively. Regarding Rose-/ECG+ group, the multivariate RR was 1.38 (0.92 to 2.08) and 2.04 (1.37 to 3.05) for men and women, respectively.

Conclusions Rose questionnaire is a powerful and independent predictor of CHD events in Iranian men and women. Although, ECG add more information beyond the other risk factors to predict $\mathrm{CHD}$ in women but in men Rose questionnaire is a better predictor than ECG.

\section{SP1-63 EVALUATION OF EARLY BODY SHAPE FOR EPIDEMIOLOGICAL RESEARCH IN ABSENCE OF OBJECTIVE MEASUREMENTS}

doi:10.1136/jech.2011.142976n.40

${ }^{1} S$ Akarolo-Anthony, ${ }^{*}{ }^{1} \mathrm{C}$ Adebamowo. ${ }^{1}$ Harvard School of Public Health, Boston, Massachusetts, USA; ${ }^{2}$ University of Maryland School of Medicine and Public Health, Baltimore, Maryland, USA

Introduction Developing countries are undergoing epidemiological transitions with increasing prevalence of non-communicable diseases (NCD). The role of early life exposure in the aetiology of many NCD's particularly the role of body shape and size for given age is increasingly appreciated. However, many environments in resource-limited settings lack early life records of birth and growth so it is not possible to correlate early life characteristics with risk of disease in these populations. Our study evaluated the use of body images as an estimator of body size and characteristics. 